\title{
Effect of reversed magnetic domains on superconductivity in $\mathrm{Pb} / \mathrm{BaFe}_{12} \mathrm{O}_{19}$ hybrids
}

\author{
Zhaorong Yang, ${ }^{\text {a) }}$ Joris Van de Vondel, Werner Gillijns, \\ Wim Vinckx, and Victor V. Moshchalkov ${ }^{\text {b) }}$ \\ Institute for Nanoscale Physics and Chemistry (INPAC), K.U. Leuven, Celestijnenlaan 200D, \\ B-3001 Leuven, Belgium \\ Ritta Szymczak \\ Polish Academy of Sciences, Institute of Physics, aleja Lotnikow 32/46, Warszawa 02-668, Poland
}

(Received 20 January 2006; accepted 28 April 2006; published online 7 June 2006)

\begin{abstract}
In this letter, the effect of reversed magnetic domains of $\mathrm{BaFe}_{12} \mathrm{O}_{19}$ on superconductivity is investigated in $\mathrm{Pb} / \mathrm{BaFe}_{12} \mathrm{O}_{19}$ hybrids. The critical field of the $\mathrm{Pb}$ film is increased by about $5 \mathrm{kOe}$ due to the compensation of the applied field by the stray field above the reversed domains. Being related to smaller critical fields of $\mathrm{Pb}$, at fields near the saturation field of $\mathrm{BaFe}_{12} \mathrm{O}_{19}$, the superconductivity can only exist above the reversed domains even at low temperatures. As a consequence of the pure reversed domain superconductivity, magnetic-field-induced superconductivity is observed in a broad temperature range. (C) 2006 American Institute of Physics. [DOI: 10.1063/1.2211167]
\end{abstract}

When the applied magnetic field exceeds a certain critical value, superconductivity is suppressed due to electromagnetic interaction and spin paramagnetic effect. Likewise, the current sent through the superconductor also generates magnetic field which can also suppress superconductivity. Practical application of superconducting materials is often limited by their small critical fields. Recently intensive research efforts have been devoted to the enhancement of the superconducting critical parameters (critical temperature $T_{c}$, field $H_{c 2}$, and current $I_{c}$ ) aimed at a broader spectrum of applications of the superconductors. ${ }^{1}$ An efficient control of the critical parameters can be achieved by using a magnetic template created by a ferromagnetic subsystem. ${ }^{2-8}$ In the superconductor/ferromagnet $(S / F)$ hybrids, inhomogeneous magnetic fields created by the ferromagnet modify quite strongly the total magnetic field in the $S$ layer, thus leading to a significant change of its superconducting properties. Using a nanoengineered lattice of magnetic $\mathrm{Co} / \mathrm{Pd}$ dots as ferromagnetic subsystem, Lange et al. observed magnetic-fieldinduced superconductivity. ${ }^{8}$ The critical field of $\mathrm{Pb}$ film was shown to increase substantially due to the compensation of the applied field in the area between the dots by the stray field of these ferromagnetic dots.

Recently, we presented the experimental observation of the domain wall superconductivity (DWS) in $\mathrm{Nb} / \mathrm{BaFe}_{12} \mathrm{O}_{19}$ hybrids. ${ }^{9}$ With the decrease of temperature in zero applied magnetic field, we found that the superconductivity first appears just above the domain walls because in that area the stray fields are the lowest. At fields near the saturation field of $\mathrm{BaFe}_{12} \mathrm{O}_{19}$, the stray field $(\sim 5 \mathrm{kOe})$ from the reversed domains compensates the applied field and favors the superconductivity to nucleate in the area above the reversed domains. The use of the reversed domain field compensator is

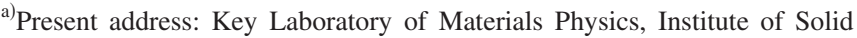
State Physics, Chinese Academy of Sciences, Hefei 230031, China; electronic mail: zryang@issp.ac.cn

b) Author to whom correspondence should be addressed; electronic mail: victor.moshchalkov@fys.kuleuven.be
}

not limited to some specific superconductors, and it should have more pronounced effects on superconductors with lower critical fields. In this letter, the modulation of superconductivity by the reversed magnetic domains is investigated in $\mathrm{Pb} / \mathrm{BaFe}_{12} \mathrm{O}_{19}$ hybrids. The critical field of the $\mathrm{Pb}$ film is increased by about $5 \mathrm{kOe}$, which is much larger than the critical field of a reference $\mathrm{Pb}$ film itself. Moreover, due to the small value of the upper critical field of $\mathrm{Pb}$, at fields near the saturation field of $\mathrm{BaFe}_{12} \mathrm{O}_{19}$, the superconductivity can exist only above the reversed domains. This is different from the $\mathrm{Nb} / \mathrm{BaFe}_{12} \mathrm{O}_{19}$ hybrids where superconductivity is present not only above reversed domains but also above positive domains at low temperatures. ${ }^{10}$ As a consequence of the pure reversed domain superconductivity, magnetic-fieldinduced superconductivity in the $\mathrm{Pb} / \mathrm{BaFe}_{12} \mathrm{O}_{19}$ hybrid system is observed in a broad temperature range.

The $\mathrm{Pb} / \mathrm{BaFe}_{12} \mathrm{O}_{19}$ samples with the $\mathrm{Pb}$ thicknesses of $d_{\mathrm{Pb}}=50 \mathrm{~nm}$ and $d_{\mathrm{Pb}}=100 \mathrm{~nm}$ have been prepared by depositing $\mathrm{Pb}$ on the single crystal $\mathrm{BaFe}_{12} \mathrm{O}_{19}$ (0001) substrates. For each sample, a $1 \mathrm{~nm}$ Ge layer was used as a buffer between $\mathrm{Pb}$ and $\mathrm{BaFe}_{12} \mathrm{O}_{19}$ and a $10 \mathrm{~nm}$ Ge cap layer was used as a protection against $\mathrm{Pb}$ oxidation. At the same time, two reference $\mathrm{Pb}$ films were evaporated on the $\mathrm{Si} / \mathrm{SiO}_{2}$ substrates under exactly the same conditions.

$\mathrm{BaFe}_{12} \mathrm{O}_{19}$ is a well-known ferrimagnet with a uniaxial anisotropy lying along its [0001] direction. The magnetic hysteresis loop of the $\mathrm{BaFe}_{12} \mathrm{O}_{19}$ (0001) substrate was measured in a Quantum Design superconducting quantum interference device (SQUID) magnetometer at $10 \mathrm{~K}$ with field perpendicular to the sample surface and is shown in Fig. 1. The loop is closed around zero field, but displays clear hysteresis around the saturation field $H_{s}$ with $H_{s}=6 \mathrm{kOe}$ and $H_{n}=4.75 \mathrm{kOe}$, where $H_{n}$ is the nucleation field. The microscopic domain structure of the $\mathrm{BaFe}_{12} \mathrm{O}_{19}$ (0001) substrate has been investigated by magnetic force microscopy (MFM) at room temperature by ramping the field from remanent state towards saturation and back again along the major hysteresis loop. ${ }^{9}$ To illustrate the evolution of the reversed domains around $H_{s}$, some of the typical MFM images are 


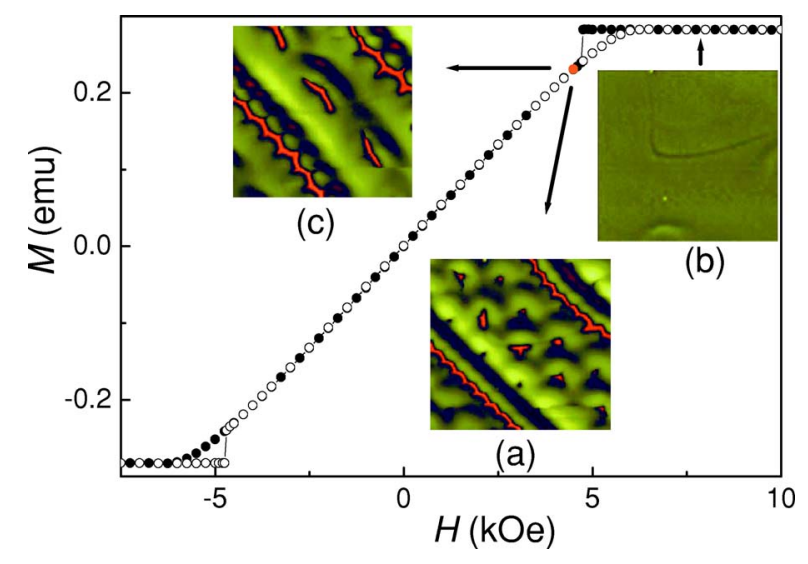

FIG. 1. (Color online) Magnetic hysteresis loop of the $\mathrm{BaFe}_{12} \mathrm{O}_{19}(0001)$ substrate. The field is first swept from -10 to $10 \mathrm{kOe}$ (open circles) and then back from 10 to $-10 \mathrm{kOe}$ (filled circles). Panels (a)-(c) show typical MFM images around the saturation field.

shown in Fig. 1. Panels (a) and (c) are recorded at the same field but with different magnetic histories. Although the magnetization loop does not show hysteresis below $H_{n}$, we can still see the difference between panels (a) and (c). If the field is applied on the way from zero towards saturation, reversed domains exist as elongated domains and interstitial bubble domains [see red areas in panel (a)]. When the field is ramped down from saturation, reversed domains exist only as elongated domains [see red areas in panel (c)] and have a total area lower than that in panel (a). Above $H_{s}$ [panel (b)], reversed domains disappear and the contrast is almost homogeneous.

The resistance $R$ of the $\mathrm{Pb} / \mathrm{BaFe}_{12} \mathrm{O}_{19}$ hybrids as well as a reference sample with $d_{\mathrm{Pb}}=100 \mathrm{~nm}$ were measured in a physical properties measurement system (Quantum Design) using a four-probe ac technique with an ac of $10 \mu \mathrm{A}$ at a frequency of $19 \mathrm{~Hz}$. The magnetic field $H$ is applied perpendicular to the sample surface from -10 to $10 \mathrm{kOe}$. From the measured $R(T)$ curves in different fixed applied fields as well as $R(H)$ curves at different fixed temperatures, we constructed magnetic field $(H)$-temperature $(T)$ phase diagrams as shown in Figs. 2(a)-2(c). The critical temperature was defined with a criterion of $R_{\text {cri }}=50 \% R_{n}$, where $R_{n}$ is the normal state resistance. For the reference film, the phase boundary displays conventional linear and symmetric (with respect to $H$ ) behavior [Fig. 2(a)]. The linear temperature dependent $T_{c}(H)$ can be fitted by $H_{c 2}(T)=\Phi_{0} / 2 \pi \xi^{2}(T)$ with $\xi(0)=42.7 \mathrm{~nm}$ and $T_{c 0}=7.23 \mathrm{~K}\left[\Phi_{0}\right.$ the superconducting flux quantum, $\xi(T)=\xi(0) /\left(1-T / T_{c 0}\right)^{1 / 2}$ the temperature dependent coherence length in the dirty limit, and $T_{c 0}$ the critical temperature at zero total field]. For the $\mathrm{Pb} / \mathrm{BaFe}_{12} \mathrm{O}_{19}$ samples, however, the $H-T$ phase boundaries are strongly asymmetric with respect to $H$, see Figs. 2(b) and 2(c). Moreover, the maximum critical temperature $T_{c}$ is shifted to around $\pm 5 \mathrm{kOe}$ and the measured value of $T_{c}$ depends on the magnetic history. For instance, the maximum of $T_{c}$ is observed at fields near $5 \mathrm{kOe}$ as the field is swept from -10 to $10 \mathrm{kOe}$, and is at negative field of $-5 \mathrm{kOe}$ if the field is swept from 10 to $-10 \mathrm{kOe}$. When the field is ramped down from $10 \mathrm{kOe}$ (or $-10 \mathrm{kOe}$ ), the superconductivity is almost suppressed near $5 \mathrm{kOe}$ (or $-5 \mathrm{kOe}$ ). In accordance with the field shift of the maximum value of $T$, the resistance as a function of field displays two dips of a different depth around $\pm 5 \mathrm{kOe}$ and shows clear hysteretic behavior [Figs. around $\pm 5 \mathrm{kOe}$ and shows clear hysteretic behavior [Figs. $5 \mathrm{kOe}$.
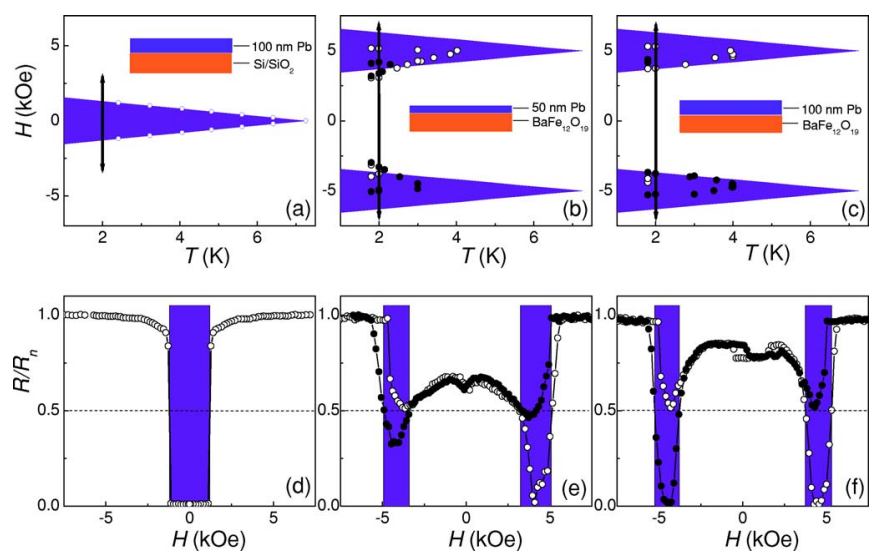

FIG. 2. (Color online) The $H$-T phase diagrams for a reference sample with $d_{\mathrm{Pb}}=100 \mathrm{~nm}$ (a), $\mathrm{Pb} / \mathrm{BaFe}_{12} \mathrm{O}_{19}$ with $d_{\mathrm{Pb}}=50 \mathrm{~nm}$ (b), and $\mathrm{Pb} / \mathrm{BaFe}_{12} \mathrm{O}_{19}$ with $d_{\mathrm{Pb}}=100 \mathrm{~nm}$ (c). The field has been applied from -10 to $10 \mathrm{kOe}$ (open circles) and back to $-10 \mathrm{kOe}$ (solid circles). Blue regions in (b) and (c) are obtained by offsetting the phase diagram of the reference sample by $5 \mathrm{kOe}$. Panels (d)-(f) show the field dependence of the normalized resistance for the reference sample, $\mathrm{Pb} / \mathrm{BaFe}_{12} \mathrm{O}_{19}$ with $d_{\mathrm{Pb}}=50 \mathrm{~nm}$, and $\mathrm{Pb} / \mathrm{BaFe}_{12} \mathrm{O}_{19}$ with $d_{\mathrm{Pb}}=100 \mathrm{~nm}$, respectively. The resistance has been measured at $2 \mathrm{~K}$ as indicated by arrows in the corresponding phase diagrams. The field is first swept from -10 to $10 \mathrm{kOe}$ (open circles) and then back from 10 to $-10 \mathrm{kOe}$ (filled circles). Blue areas correspond to the superconducting state.

2(e) and 2(f)]. In contrast to that, the $R(H)$ curve for the reference sample [Fig. 2(d)] is symmetric with respect to $H$ and does not depend on the magnetic history.

In the present system, the field shift of the maximum $T_{c}$ value and the hysteretic resistance transition can be understood by taking into account the domain evolution in $\mathrm{BaFe}_{12} \mathrm{O}_{19}$. In the $\mathrm{Pb} / \mathrm{BaFe}_{12} \mathrm{O}_{19}$ hybrids, inhomogeneous stray fields created by $\mathrm{BaFe}_{12} \mathrm{O}_{19}$ modify substantially the total internal magnetic field in the $\mathrm{Pb}$ layer and thus form a tunable magnetic template for the nucleation of superconductivity. Above $H_{s}$, the total magnetic field in the $\mathrm{Pb}$ layer is strengthened by the stray field from the substrate and is larger than the upper critical field $H_{c 2}$. The $\mathrm{Pb}$ film is therefore in the normal state. Below $H_{n}$, positive domains and reversed domains exist separated by magnetic domain walls. The reversed domains, with their stray field $H_{d}(\sim 5 \mathrm{kOe})$ opposite to the applied field $H_{a}$, are practically the field compensators for the superconductor. Corresponding to almost complete field compensation at fields near $5 \mathrm{kOe},\left|H_{a}-H_{d}\right|$ $\approx 0$, the maximum value of $T_{c}$ is observed in this field region. As a result of the appearance of superconductivity above the reversed domains, the resistance drops abruptly and the measured magnitude depends on the total area of the available reversed domains. ${ }^{9}$ Following the hysteretic domain evolution, for field sweeping up and down, a hysteresis also appears in the $R(H)$ curves. As the field is ramped down towards zero, the difference in amplitudes between $\left|H_{d}\right|$ and $\left|H_{a}\right|$ grows and the field compensation effect above the reversed domains becomes less efficient. However, at the domain wall area the stray fields are substantially lower and there a weak field compensation effect is still possible. As shown in Figs. 2(e) and 2(f), the resistance at low fields is less than the normal state resistance, suggesting the existence of weak superconductivity above domain wall areas. This remarkable transformation of the superconductivity area with the applied field is responsible for the appearance of the two minima of a different depth in the $R(H)$ curves around

to AIP license or copyright; see http://apl.aip.org/apl/copyright.jsp 
Based on almost the same explanation as we gave for the $\mathrm{Nb} / \mathrm{BaFe}_{12} \mathrm{O}_{19}$ hybrids, the field shift of the maximum value of $T_{c}$ and the hysteretic resistance transition in the $\mathrm{Pb} / \mathrm{BaFe}_{12} \mathrm{O}_{19}$ can be understood. However, it should be mentioned that, due to the difference in upper critical fields of $\mathrm{Pb}$ and $\mathrm{Nb}$, the effect of the magnetic template created by $\mathrm{BaFe}_{12} \mathrm{O}_{19}$ on the superconductivity is different. For the $\mathrm{Nb} / \mathrm{BaFe}_{12} \mathrm{O}_{19}$, because of a larger value of $H_{c 2}$, the constraint of $\left|H_{a}\right|+\left|H_{d}\right|<H_{c 2}$ can be fulfilled even in the areas above the positive domains at sufficiently lower temperatures. ${ }^{10}$ Due to the formation of bulk superconductivity, reversed domain superconductivity and domain wall superconductivity can be distinguished only in a narrow temperature range (less than $1 \mathrm{~K}$ ). At lower temperatures, both the resistance dips and the hysteretic resistance transition disappear. In contrast to that, in the $\mathrm{Pb} / \mathrm{BaFe}_{12} \mathrm{O}_{19}$ hybrids, at fields near the saturation field of $\mathrm{BaFe}_{12} \mathrm{O}_{19}$, superconductivity can only exist above the domains with polarity opposite to the applied field. Even for ideal field compensation [see Figs. 2(b) and 2(c)] upper and lower superconducting regions (blue areas) on the $H-T$ diagram do not overlap due to the smaller upper critical fields of the reference $\mathrm{Pb}$ film. If the field is swept up and down at a fixed temperature, superconductivity is always induced around $5 \mathrm{kOe}$ [blue areas in Figs. 2(e) and 2(f)], provided the temperature is below the value of $T_{c}(H=0)$.

In conclusion, the effect of reversed magnetic domains on superconductivity is studied in the $\mathrm{Pb} / \mathrm{BaFe}_{12} \mathrm{O}_{19}$ hybrids. We clearly have demonstrated that the magnetic template created by $\mathrm{BaFe}_{12} \mathrm{O}_{19}$ is more effective for the field compensation effects in superconductors with smaller critical fields.
In these superconductors, the critical field has been enhanced to a remarkably high value, which has also led to the appearance of a broad temperature window for the realization of the field-induced superconductivity. This result is important for designing logical devices based on the superconductivity manipulation with the help of switchable magnetic domains of different polarity.

The authors would like to thank A. Volodin for the MFM measurements. This work is supported by the KU Leuven Research Fund GOA/2004/02, Flemish FWO, Belgian IUAP, and Bilateral Flanders-China Project BIL 04/03 Programmes.

${ }^{1}$ V. V. Moshchalkov, V. Bruyndoncx, L. Van Look, M. J. Van Bael, Y. Bruynseraede, and A. Tonomura, Handbook of Nanostructured Materials and Nanotechnology (Academic, San Diego, 2000), Vol. 3, Chap. 9, p. 451.

${ }^{2}$ For recent reviews see I. F. Lyuksyutov and V. L. Pokrovsky, Adv. Phys. 54, 67 (2005); A. I. Buzdin, Rev. Mod. Phys. 77, 935 (2005).

${ }^{3}$ I. F. Lyuksyutov and V. L. Pokrovsky, Phys. Rev. Lett. 81, 2344 (1998).

${ }^{4}$ S. Erdin, I. F. Lyuksyutov, V. L. Pokrovsky, and V. M. Vinokur, Phys. Rev. Lett. 88, 017001 (2002)

${ }^{5}$ J. I. Martn, M. Vlez, J. Nogus, and I. K. Schuller, Phys. Rev. Lett. 79, 1929 (1997).

${ }^{6}$ L. N. Bulaevskii, E. M. Chudnovsky, and M. P. Maley, Appl. Phys. Lett. 76, 2594 (2000).

${ }^{7}$ D. S. Golubovic, W. V. Pogosov, M. Morelle, and V. V. Moshchalkov, Appl. Phys. Lett. 83, 1593 (2003).

${ }^{8}$ M. Lange, M. J. Van Bael, Y. Bruynseraede, and V. V. Moshchalkov, Phys. Rev. Lett. 90, 197006 (2003).

${ }^{9}$ Z. R. Yang, M. Lange, A. Volodin, R. Szymczak, and V. V. Moshchalkov, Nat. Mater. 3, 793 (2004)

${ }^{10}$ Z. R. Yang, R. Szymczak, K. Vervaeke, and V. V. Moshchalkov, Phys. Rev. B (to be published). 